\title{
The Binding Problem after an eye movement
}

\author{
Emma Wu Dowd ${ }^{1}$ (D) - Julie D. Golomb ${ }^{1}$
}

Published online: 3 June 2019

(C) The Psychonomic Society, Inc. 2019

\begin{abstract}
Spatial attention is thought to be the "glue" that binds features together (e.g., Treisman \& Gelade, 1980, Psychology, 12[1], 97136) - but attention is dynamic, constantly moving across multiple goals and locations. For example, when a person moves her eyes, visual inputs that are coded relative to the eyes (retinotopic) must be rapidly updated to maintain stable world-centered (spatiotopic) representations. Here, we examined how dynamic updating of spatial attention after a saccadic eye movement affects object-feature binding. Immediately after a saccade, participants were simultaneously presented with four colored and oriented bars (one at a precued spatiotopic target location) and instructed to reproduce both the color and orientation of the target item. Object-feature binding was assessed by applying probabilistic mixture models to the joint distribution of feature errors: feature reports for the target item could be correlated (and thus bound together) or independent. We found that compared with holding attention without an eye movement, attentional updating after an eye movement produced more independent errors, including illusory conjunctions, in which one feature of the item at the spatiotopic target location was misbound with the other feature of the item at the initial retinotopic location. These findings suggest that even when only one spatiotopic location is task relevant, spatial attention - and thus object-feature binding - is malleable across and after eye movements, heightening the challenge that eye movements pose for the binding problem and for visual stability.
\end{abstract}

Keywords Eye movements and visual attention · Attention: space-base

"Where's my coffee mug? Did I grab the right set of keys? One, two, three kids, all here!" Object recognition is a crucial part of everyday life, yet visual objects are each composed of multiple visual features (e.g., color, shape, texture) that must

\begin{abstract}
Significance Visual object perception requires integration of multiple features, and according to Treisman's feature integration theory, spatial attention is critical for object-feature binding. But attention is dynamic, especially when our eyes are moving. Here, we examined how the updating of spatial attention across saccadic eye movements - and its consequences for how attention is deployed - affects visual object integrity. Probabilistic mixture modeling revealed more independent errors, including illusory conjunctions, immediately after a saccade. This work has important implications for our understanding of how spatial attention is remapped across eye movements, as well as the broader challenge of object integrity and visual stability.
\end{abstract}

Electronic supplementary material The online version of this article (https://doi.org/10.3758/s13414-019-01739-y) contains supplementary material, which is available to authorized users.

Emma Wu Dowd

emma.wu.dowd@austin.utexas.edu

1 Department of Psychology, The Ohio State University, 1835 Neil Ave, Columbus, OH 43210, USA be processed and integrated together into a cohesive objectlevel representation (i.e., "the binding problem"; Treisman, 1996). The crux of Anne Treisman's feature integration theory (Treisman \& Gelade, 1980) is that spatial attention serves as the "glue" that binds features together. The idea is that features falling within the same spatial window of attention are grouped together into an integrated object (e.g., Duncan, 1984; O'Craven, Downing \& Kanwisher, 1999; Schoenfeld et al., 2003). Consequently, conditions of limited attention can lead to failures of object-feature binding (i.e., binding between multiple feature dimensions), as evidenced by "illusory conjunctions," such as viewing a green square and a red circle, but reporting a green circle (Treisman \& Schmidt, 1982; see also Wolfe \& Cave, 1999). The strong consensus is that the ability to maintain a precise spatial focus of attention is critical for preserving object integrity (Nissen, 1985; Pertzov \& Husain, 2013; Reynolds \& Desimone, 1999; Schneegans \& Bays, 2017; Treisman \& Gelade, 1980).

Visual attention, however, is rarely singular or static. In the real world, multiple objects with multiple features are simultaneously present in the environment, and attention is constantly moving across multiple goals and locations. Previous work has demonstrated striking errors of binding under 
dynamic conditions of spatial attention (Dowd \& Golomb, 2019; Golomb, 2015; Golomb, L'Heureux, \& Kanwisher, 2014). In one set of studies (Golomb et al., 2014), participants were presented with an array of simple, single-feature objects (i.e., colored squares) and were asked to reproduce the color of a target item (i.e., continuous-report paradigm; Wilken \& Ma, 2004). Covert spatial attention was cued to shift from one location to another, to split across two locations simultaneously, or to hold stable across an eye movement. Probabilistic mixture modeling (e.g., Bays, Catalao, \& Husain, 2009; Zhang \& Luck, 2008) showed that shifting attention produced more "swap errors" (i.e., misreporting a nontarget color; see Bays, 2016), whereas splitting attention resulted in a blending between the two attended colors (Golomb et al., 2014). Intriguingly, holding covert attention across an eye movement produced both types of errors (discussed below). More recently, we extended this paradigm to probe multifeature objects, using a joint continuous report (i.e., reproduce both the color and orientation of a target) and joint probabilistic modeling to fit responses from multiple feature dimensions simultaneously (Dowd \& Golomb, 2019; see also Bays, Wu, \& Husain, 2011). We found that splitting attention across multiple locations degraded object integrity (e.g., reporting the color of the target, but an incorrect orientation), while rapid shifts of spatial attention maintained bound objects, even when reporting the wrong object altogether (e.g., reporting both the color and the orientation of the swapped object).

In the current study, we focus on a special case of dynamic attention: saccadic eye movements. Eye movements pose a unique challenge to visual stability because visual inputs are coded relative to the eyes, in "retinotopic" coordinates, which are constantly moving - yet we perceive and act upon stable world-centered "spatiotopic" representations. Thus, retinotopic information must be rapidly updated with each eye movement. It has been suggested that neurons may "remap" their receptive fields in anticipation of a saccade (Duhamel, Colby, \& Goldberg, 1992), and spatial attention can also shift predictively to a remapped location (Rolfs, Jonikaitis, Deubel, \& Cavanagh, 2011). However, remapping of spatial attention may not be as rapid or efficient as it seems. In addition to "turning on" the new location, there seems to be a second stage of "turning off" the previous location: For a brief window of time after each eye movement, spatial attention temporarily lingers at the previous retinotopic location (the "retinotopic attentional trace"; Golomb, Nguyen-Phuc, Mazer, McCarthy, \& Chun, 2010; Talsma, White, Mathôt, Munoz, \& Theeuwes, 2013) before updating to the correct spatiotopic location (e.g., Golomb, Chun, \& Mazer, 2008; Mathôt \& Theeuwes, 2010; see also Jonikaitis, Szinte, Rolfs, \& Cavanagh, 2013). The idea is that attention can be allocated to a new retinotopic location before it disengages from the previous retinotopic location, resulting in a transient period in which both locations are simultaneously attended
(Golomb, Marino, Chun, \& Mazer, 2011; see also Khayat, Spekreijse, \& Roelfsema, 2006).

Like dynamic shifts and splits of covert spatial attention, dynamic remapping of spatial attention across saccades also induces errors of feature perception: Golomb et al. (2014) included a condition where participants were instructed to reproduce the color of an item appearing at a spatiotopic target location at different delays after a saccade; the target location was cued before the saccade, so that the retinotopic coordinates needed to be remapped with the saccade. Critically, a different nontarget color was simultaneously presented at the retinotopic trace location. When the color array was presented at a short delay following the saccade $(50 \mathrm{~ms})$, there were two distinct types of errors: On some trials, participants made swap errors and misreported the color of the retinotopic distractor instead of the target - as if spatial attention was stuck at the presaccadic retinotopic location instead of updating to the spatiotopic location. More intriguingly, on other trials, color reports were systematically shifted toward the color of the retinotopic distractor (similar to the blending seen in the split attention condition of the same study), consistent with the premise that spatial attention was temporarily split between the two locations during remapping. Importantly, at longer postsaccadic delays (500 $\mathrm{ms}$ ), subjects accurately reported the spatiotopic color. Together, these results suggest that that even incidental and residual spatial attention after an eye movement (i.e., the retinotopic trace) is sufficient to distort feature representations. How then does dynamic remapping of spatial attention impact visual object integrity?

Here, we examine object-feature binding during the crucial postsaccadic period of dynamic attentional remapping. As in Dowd and Golomb (2019), we asked participants to reproduce multiple features at a target location, allowing us to assess object-feature binding by modeling responses from multiple feature dimensions simultaneously (see also Bays et al., 2011). However, while our previous paper manipulated dynamic spatial attention by cueing different spatial locations while the eyes remained fixated, the current study examines dynamic remapping of attention induced by a saccadic eye movementcomparing performance at different delays after a saccade to performance with no saccade at all. In other words, we combined the saccadic remapping manipulation of Golomb et al. (2014) with the multifeature design of Dowd and Golomb (2019). We predicted that if attentional remapping results in a transient splitting of spatial attention across the spatiotopic target location and the retinotopic trace location, object integrity should degrade during this period. However, when spatial attention does not need to remap (i.e., no saccade) or has completely updated after a saccade, object integrity should be maintained. Evidence for transient "unbinding" of visual objects would imply that spatial attention-and thus objectfeature binding - is malleable across and after eye movements, heightening the challenge of visual stability. 


\section{Method}

Our participant recruitment techniques, target sample size, exclusion rules, stimuli, task design and procedure, and statistical models and analyses were preregistered on the Open Science Framework (http://osf.io/y495a) prior to data collection.

\section{Participants}

Data from 25 participants (ages 18-34 years; 17 female) from The Ohio State University were included in the final analyses, according to our preregistered power analyses. Preregistered exclusion criteria required that each participant complete at least 10 blocks ( 320 trials) across two 1-hour experimental sessions. Preregistered exclusion criteria also required that each participant's $p T_{C} T_{O}$ parameter estimate (as explained below) be greater than 0.5 , as an indication that they were performing the task correctly, but no subject's data were excluded for this reason. However, there was one more volunteer whose data resulted in $p T_{C} T_{O}>0.5$, but their associated estimates of feature precision $(\sigma)$ were $>2.5$ standard deviations from all other participants - indicating poor performance and making it difficult to interpret the other model parameters for this subject. (In the current model, $p T_{C} T_{O}$ is directly affected by precision, such that an extremely large $\sigma$ parameter may inflate estimates of $p T_{C} T_{O}$.) Thus, we also excluded the data from this volunteer; this exclusion criterion was not preregistered. All participants reported normal or correctedto-normal visual acuity and color vision, received course credit or $\$ 10 /$ hour, and provided informed consent in accordance with The Ohio State University Institutional Review Board.

\section{Stimuli and procedure}

Stimuli were presented on a 21-inch flat-screen ViewSonic Graphic Series G225f CRT monitor with a refresh rate of $85 \mathrm{~Hz}$ and screen resolution of $1,280 \times 1,024$ pixels, using MATLAB and the Psychophysics Toolbox extensions (Kleiner, Brainard, \& Pelli, 2007). Subjects were positioned with a chin rest approximately $60 \mathrm{~cm}$ from the monitor in a dimly lit room. Eye position was monitored with a desktop-mounted EyeLink 1000 eye-tracking system and the Eyelink Toolbox (Cornelissen, Peters, \& Palmer, 2002), which were calibrated using a ninepoint grid procedure and sampled observers' left eyes at 500 Hz. No drift corrections were used, but participants were recalibrated between blocks as needed. The monitor was color calibrated with a Minolta CS-100 colorimeter.

Figure 1a illustrates an example trial sequence. Each trial began with the presentation of a white fixation dot (diameter of $0.6^{\circ}$ ) presented at one of four locations on the screen (the corners of an imaginary $10.5^{\circ} \times 10.5^{\circ}$ square; see Fig. 1b). Once participants had accurately fixated for $1,000 \mathrm{~ms}$, as determined by real-time eye tracking, the trial continued as follows:

A single spatial black cue (black $4^{\circ} \times 4^{\circ}$ square outline, stroke width $\left.=0.1^{\circ}\right)$ was presented $\left(7.4^{\circ}\right.$ eccentricity from fixation) for $500 \mathrm{~ms}$. Participants were instructed to covertly attend to the cued location. After another 1,000-ms fixation period, on half of the trials the fixation dot jumped to a horizontally or vertical adjacent position. On these saccade trials, subjects had to immediately move their eyes to the new location. On the other half of trials (no-saccade trials), the fixation dot remained at the original location, and subjects held fixation for a similar amount of time based on average saccadic latency from a previous experiment (350 ms; Golomb et al., 2014; see also Supplement S3). Both the location of the cue (five possible locations on the screen,; see Fig. 1b) and the presence and direction of the saccade were randomized.

After a delay of either $50 \mathrm{~ms}$ or $500 \mathrm{~ms}^{1}$ from the time of successful saccade completion (as determined by real-time eye tracking), an array of four colored and oriented bars $\left(0.75^{\circ} \times 4^{\circ}\right)$ appeared at equidistant locations around fixation ( $7.4^{\circ}$ eccentricity) for $50 \mathrm{~ms}$. One of these stimuli appeared at the same spatiotopic (absolute) location of the cue - this was the "target" (T) that subjects were supposed to report. On saccade trials, another stimulus occupied the same retinotopic location (relative to the eyes) as the cue - this was the critical nontarget (N1). The other adjacent nontarget (N2) and the diagonal nontarget (N3) were considered control items. In no-saccade trials, the cued location was both spatiotopic and retinotopic, so "N1" and "N2" were arbitrarily assigned to the stimuli adjacent to the target.

The color of the target item was chosen randomly on each trial from 180 possible colors, which were evenly distributed along a $360^{\circ}$ circle in CIE $L^{*} a^{*} b^{*}$ coordinates with constant luminance ( $L^{*}=70$, center at $a^{*}=20, b^{*}=38$, and radius 60 ; Zhang \& Luck, 2008). The colors of the remaining stimuli were chosen so that the adjacent items (N1 and N2) were equidistant in opposite directions $\left(90^{\circ}\right.$ clockwise or counterclockwise deviation along the color wheel, with direction randomly varying from trial to trial), and the item at the diagonal location (N3) was set $180^{\circ}$ away in color space. The orientation of the target item was also chosen randomly on each trial from a range of angles $0^{\circ}-180^{\circ}$, and $\mathrm{N} 1$ and $\mathrm{N} 2$ were likewise equidistant in opposite directions ( $45^{\circ}$ clockwise or counterclockwise deviation), with N3 set $90^{\circ}$ away. Feature values for color and orientation were set independently, as was the direction of deviation for each feature. The stimulus array was followed by $200 \mathrm{~ms}$ of masks (squares colored with a random color value at each pixel location, covering each of the four stimulus locations).

\footnotetext{
${ }^{1}$ Because of the screen refresh rate $(85 \mathrm{~Hz})$, actual presentation durations were a few ms shorter. For instance, a 50-ms duration was actually $47 \mathrm{~ms}$, and a $500-$ ms duration was actually $496 \mathrm{~ms}$. Stimulus presentation durations have been rounded up for consistency with previously published experiments (e.g., Dowd \& Golomb, 2019; Golomb, 2015; Golomb et al., 2014).
} 
a

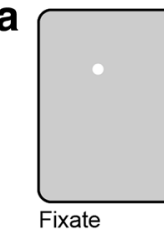
$1000 \mathrm{~ms}$

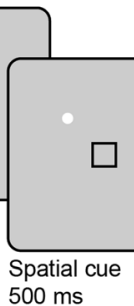

Fig. 1 a Example trial sequence for a saccade trial. Participants were cued to covertly attend to a spatial precue and reproduce both the color and orientation (i.e., joint continuous report) of whichever stimulus subsequently appeared at that cued (spatiotopic) location. On nosaccade trials, participants maintained fixation at a single location across the trial. On saccade trials, the fixation dot moved to a new location prior to stimulus presentation, and participants had to accurately make a saccade to the new fixation location. The array appeared at either $50 \mathrm{~ms}$ or $500 \mathrm{~ms}$ after completion of the saccade, followed by a mask array. For the joint-feature report, participants adjusted a probe bar presented at fixation

Participants then made a joint continuous-report response, reporting the color and orientation of the target item. A single probe bar with random initial values for color and orientation was presented at fixation (i.e., the second fixation location in saccade trials). Participants were instructed to adjust the color and orientation of the probe item to match the features of the target. The probe's features were adjusted using two sets of adjacent keys on either side of same keyboard: $[\mathrm{z}]$ and $[\mathrm{x}]$ (left-color) and $[,<]$ and $[.>]$ (right-orientation). Pressing down on one set of keys caused the probe to rotate through the $180^{\circ}$ range of possible orientations (steps of $1^{\circ} ;[.>]$ clockwise, $[,<]$ counterclockwise); pressing down on the other set of keys caused the probe's color to cycle through the $360^{\circ}$ space of possible colors (steps of $2^{\circ}$; $[\mathrm{x}]$ clockwise, $[\mathrm{z}]$ counterclockwise). Participants could adjust the two features in any order. To input their response, participants pressed the space bar. Accuracy was stressed, and there was a time limit of $10 \mathrm{~s}$.

Then participants also made a four-alternative forcedchoice location response: Four location placeholders (white $4^{\circ} \times 4^{\circ}$ square outlines, stroke width $=0.1^{\circ}$ ) were displayed at the four stimulus locations around fixation. Pressing the right-hand set of keys rotated through each location and highlighted the placeholder with a thicker white outline $\left(\right.$ stroke width $\left.=0.3^{\circ}\right)$. Participants were instructed to select the cued (spatiotopic) location and input their response by pressing the space bar, with a time limit of $5 \mathrm{~s}$. b

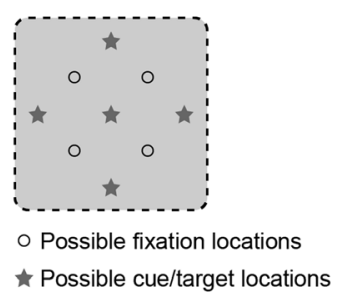

to match the color and orientation of the target item. After the joint-feature report, participants additionally reported the location of the target item. In this example, the target item is the upper-right yellow bar, marked "T," and the critical N1 nontarget is the lower-right green bar, marked "N1" (gray labels shown in the example are illustrative only and were not displayed during the actual task). On saccade trials, the critical N1 nontarget is the item which appears at the same retinotopic location as the initial spatial cue (i.e., relative to fixation). b In this task, there were four possible fixation locations (open circles) and five possible target locations (gray stars)

At the end of the trial, participants were shown feedback for 1,500 ms: The reported color-orientation response was shown at fixation, and the actual target item was displayed in its original location. The reported location was displayed as a white outline on the same screen.

At any point in the trial, if the subject's eye position deviated more than $2^{\circ}$ from the correct fixation location, or if saccadic latency was greater than $600 \mathrm{~ms}$, the trial was immediately aborted and repeated later in the block. All participants completed 10-16 blocks (as time permitted) of 32 intermixed $2 \times 2$ (saccade vs. no-saccade, early vs. later) trials, resulting in 80-128 trials of each saccade-delay condition, across two separate experimental sessions. Only the first session began with fixation training and practice trials (six no-saccade, 12 saccade trials). Trials were discarded if subjects made no color and no orientation adjustments before inputting their response $(<0.1 \%)$. For nosaccade trials, the delay manipulation should have, in practice, made no difference; with no saccade being triggered, the array simply appeared $1,400 \mathrm{~ms}$ or $1,850 \mathrm{~ms}$ after the offset of the spatial cue. Indeed, there was no statistically significant difference in $p T_{C} T_{O}$ (as explained below) when no-saccade-early and no-saccade-later trials were analyzed separately. Thus, no-saccade trials were collapsed across early and later delays and analyzed as a single condition for all subsequent analyses. 


\section{Joint-feature analyses}

On each trial, response error was calculated as the angular deviation between the continuous probe report and the cued target item, for each feature separately $\left(\theta_{C}=\right.$ color error, range: $-180^{\circ}$ to $180^{\circ} ; \theta_{O}=$ orientation error, range: $-90^{\circ}$ to $\left.90^{\circ}\right)$. For Saccade trials, although the direction of N1 varied randomly in relation to $\mathrm{T}$ (clockwise or counterclockwise in terms of color or orientation space), we aligned the responses on each trial so that errors toward the $\mathrm{N} 1$ feature were always coded as positive deviations $\left(+90^{\circ}\right.$ or $\left.+45^{\circ}\right)$, and errors toward N2 as negative deviations $\left(-90^{\circ}\right.$ or $\left.-45^{\circ}\right)$.

To quantify the amount of object-feature binding, we adopted the same mixture modeling approach as in Dowd and Golomb (2019; as based on Bays et al., 2009; Bays et al., 2011; Golomb et al., 2014; Zhang \& Luck, 2008). Within each single feature dimension, responses could be attributed to either reporting the target ( $T$, a von Mises distribution centered on the target feature value), misreporting the critical retinotopic nontarget ( $N 1$, a von Mises distribution centered on the $\mathrm{N} 1$ feature value), or random guessing ( $U$, a uniform distribution across all feature values). Our focus here was on dynamic remapping of spatial attention from the retinotopic N1 location to the spatiotopic T location; thus, because responses to $\mathrm{N} 2$ and $\mathrm{N} 3$ were theoretically less relevant, our model of interest does not specify separate distributions centered on those items. In this model, what few responses do occur to $\mathrm{N} 2$ and $\mathrm{N} 3$ would be absorbed by $U$ (see Supplement S1 for additional information and an alternative model incorporating all of these parameters).

Critically, we modeled color and orientation as joint probability distributions, fitting responses from both feature dimensions simultaneously (Bays et al., 2011; Dowd \& Golomb, 2019). In the simple joint model, we modeled the three types of feature reports described above ( $T, N 1$, and $U$ ) for each dimension, resulting in nine response combinations of color (three) and orientation (three), plus four parameters for the concentrations $\mathrm{K}_{\mathrm{C}}$ and $\mathrm{\kappa}_{\mathrm{O}}(\sigma=\sqrt{1 / \kappa})$ and means $\mu_{\mathrm{C}}$ and $\mu_{\mathrm{O}}$ of the target. The joint distribution of responses was thus modeled as:

$p\left(\theta_{C}, \theta_{O}\right)=\sum_{m} \alpha_{m} p_{m}$,

where $\theta_{C}$ and $\theta_{O}$ are the reported feature errors; $m$ is the number of joint color-orientation response combinations, with $m_{\text {Simple }}=1: 9 ; \alpha_{m}$ is the probability of each response combination; and $p_{m}$ represents the joint probability density distribution for that combination. Table 1 lists each of the nine combinations and associated probability density functions. For example, the joint probability distribution of reporting the target color and the N1 nontarget orientation would be $p T_{C} N 1_{O}=\phi_{\mu_{C}, \kappa_{C}} \phi_{\frac{\pi}{2}, \kappa_{O}}$.

Joint-feature response distributions were fit using Markov chain Monte Carlo (MCMC), as implemented through custom MATLAB scripts (available on Open Science Framework) using the MemToolbox (Suchow, Brady, Fougnie, \& Alvarez, 2013) on the Ohio Supercomputer Center (Ohio Supercomputer Center, 1987). The MCMC procedure sampled three parallel chains across as many iterations as necessary to achieve convergence, according to the method of Gelman and Rubin (1992). We collected 15,000 postconvergence samples and used the posterior distributions to compute the maximum-likelihood estimates of each parameter, as well as its $95 \%$ highest posterior density interval (HDI). For our primary analyses using the simple model, we adopted a standard within-subjects analytical approach: Parameter estimates were obtained separately for each individual subject and each trial type, then evaluated with frequentist significance testing. Post hoc tests were evaluated with the appropriate Bonferroni correction for multiple comparisons. As a supplementary analysis, we also fit the data using a full joint model, which includes mixtures of $T, N 1$, $N 2, N 3$, and $U$; more details can be found in the Supplemental Methods and Results.

Table 1 Simple joint model response distributions combined across both nonspatial feature dimensions

\begin{tabular}{|c|c|c|c|c|}
\hline & Response type & $m_{\text {Simple }}$ & Response combination & Joint probability density \\
\hline \multirow[t]{2}{*}{ Correlated } & Correlated target & 1 & $T_{C} T_{O}$ & $\phi_{\mu_{C}, \mathrm{~K}_{C}} \phi_{\mu_{O}, \mathrm{~K}_{O}}$ \\
\hline & Correlated swap & 2 & $N 1_{C} N 1_{O}$ & $\phi_{\frac{\pi}{2}, K_{C}} \phi_{\frac{\pi}{4}, K_{O}}$ \\
\hline \multirow[t]{6}{*}{ Independent } & Illusory conjunction & 3 & $T_{C} N 1_{O}$ & $\phi_{\mu_{C}, \mathrm{~K}_{C}} \phi_{\frac{\pi}{4}, \mathrm{~K}_{O}}$ \\
\hline & & 4 & $N 1_{C} T_{O}$ & $\phi_{\frac{\pi}{2}, \kappa_{C}} \phi_{\mu_{O}, \kappa_{O}}$ \\
\hline & Unbound target & 5 & $T_{C} U_{O}$ & $\phi_{\mu_{C},{ }_{C}} \gamma_{O}$ \\
\hline & & 6 & $U_{C} T_{O}$ & $\gamma_{C} \phi_{\mu_{O}, \kappa_{O}}$ \\
\hline & Unbound nontarget & 7 & $N 1_{C} U_{O}$ & $\phi_{\frac{\pi}{2}, \kappa_{C}} \gamma_{O}$ \\
\hline & & 8 & $U_{C} N 1_{O}$ & $\gamma_{C} \phi_{\frac{\pi}{4}, \mathrm{~K}_{O}}$ \\
\hline Random guessing & & 9 & $U_{C} U_{O}$ & $\gamma_{C} \gamma_{O}$ \\
\hline
\end{tabular}

Note. For the current experiment, the simple model was restricted to the spatiotopic T and critical retinotopic N1 items, given our theoretical focus on remapping spatial attention 


\section{Results}

By probing both color and orientation on each trial, we examined whether errors in recalling multiple features of the same object were correlated (and thus bound together) or independent (and unbound) immediately after an eye movement. Figure 2 visualizes the joint distribution of responses by plotting individual trials in joint-feature space, where the vertical and horizontal axes correspond to the color and orientation errors, respectively.

"Object integrity" was inferred from contrasting correlated responses (i.e., reporting both the color and orientation of the same item) with independent responses (i.e., reporting only one feature of an item; see Table 1 and Fig. 2a for predicted distributions). Correlated target responses thus refer to reporting both features of the correct target item $\left(\mathrm{T}_{\mathrm{C}} \mathrm{T}_{\mathrm{O}}\right)$, which would be represented as a two-dimensional Gaussian density centered on the origin $\left(0^{\circ}\right.$ error). Correlated $\mathrm{N}_{\mathrm{C}} \mathrm{N} 1_{\mathrm{O}}$ "swap" errors refer to misreporting both features of the retinotopic N1 distractor, which would be represented as a two-dimensional Gaussian density on the positive-slope diagonal of joint-feature space. Failures in object-feature binding, on the other hand, would result in independent responses, such as reporting the color of an item without also reporting the orientation of the same item. Independent errors could be due to reporting only one feature of the target and guessing the other (unbound target; e.g., $\mathrm{T}_{\mathrm{C}} \mathrm{U}_{\mathrm{O}}$ ); reporting only one feature of the $\mathrm{N} 1$ nontarget and guessing the other (unbound N1; e.g., $\mathrm{N}_{\mathrm{C}} \mathrm{U}_{\mathrm{O}}$ ); or misbinding the features of the target and the $\mathrm{N} 1$ nontarget (illusory conjunction; e.g., $\mathrm{T}_{\mathrm{C}} \mathrm{N} 1_{\mathrm{O}}$ ). For instance, in joint-feature space, independent target errors are represented as a distribution of responses along the horizontal or vertical axes of joint-feature space (i.e., centered on zero error in one dimension but not the other; see Fig. 2a).

Figure $2 \mathrm{~b}$ presents scatterplots of the empirical data, collapsed across all participants, for no-saccade, saccade-early, and saccade-later trials. We quantified the different response types with joint-feature probabilistic models, and the corresponding parameter estimates from the simple model are summarized in Table 2 and Fig. 3.

\section{a Predicted Distributions}
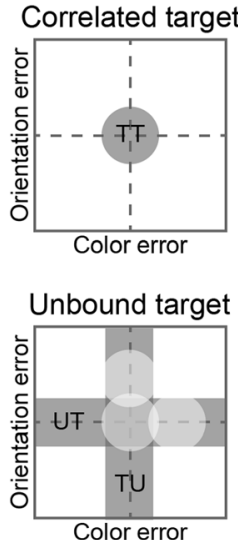

Correlated N1N1 swap
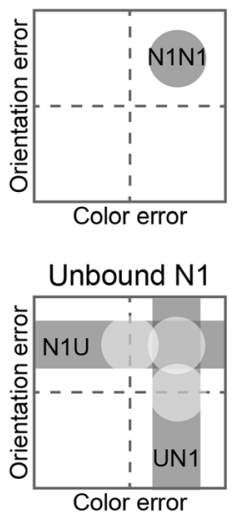

b Empirical Distributions

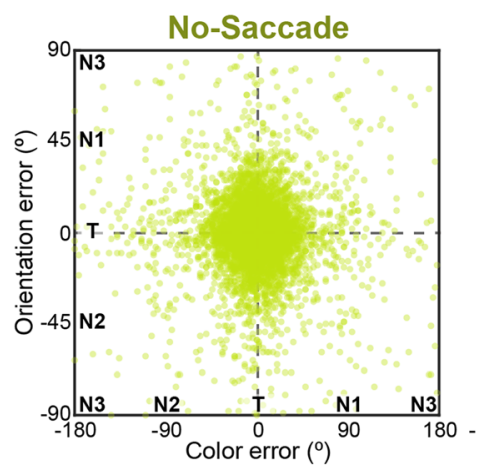

Fig. 2 Visualizations of color-orientation reports in joint-feature space, plotted as error relative to actual target feature values: color responses are shown along the $x$-axis, and orientation responses are shown along the $y$ axis. Dashed lines at the origin indicate $0^{\circ}$ error. For visualization purposes, we have flattened joint-feature space; both feature dimensions are in fact circular, such that $+180^{\circ}$ is identical to $-180^{\circ}$ in color space. The

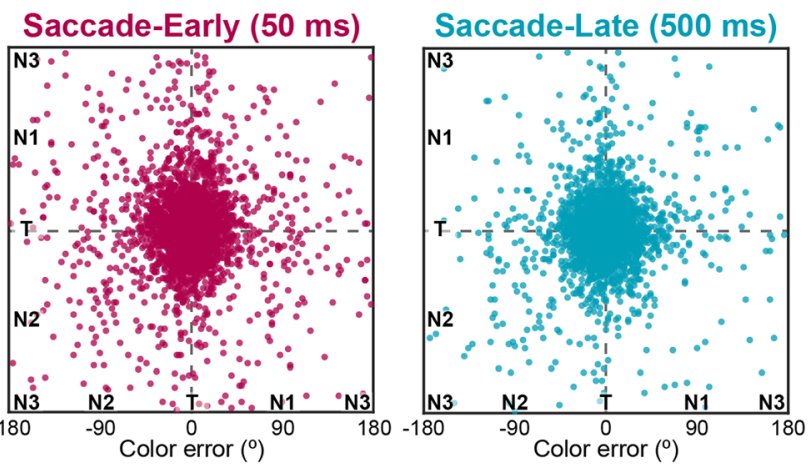

schematics in (a) show predicted distributions for possible response types, with correlated responses on the top row and independent responses on the bottom row. The scatterplots in (b) plot trial-by-trial empirical error distributions separately for no-saccade, saccade-early, and saccade-late trials. Each dot represents the corresponding color and orientation response for a single trial, aggregating across subjects 
Table 2 Simple joint model parameter estimates $(N=25)$

\begin{tabular}{llll}
\hline & No saccade & $\begin{array}{l}\text { Saccade- } \\
50 \text {-ms delay }\end{array}$ & $\begin{array}{l}\text { Saccade- } \\
500 \text {-ms delay }\end{array}$ \\
\hline$p T_{C} T_{O}$ & $.864(.131)$ & $.802(.164)$ & $.864(.117)$ \\
$p N 1_{C} N 1_{O}$ & $.005(.007)$ & $.005(.006)$ & $.006(.008)$ \\
$p T_{C} N 1_{O}$ & $.013(.018)$ & $.017(.018)$ & $.013(.017)$ \\
$p N 1_{C} T_{O}$ & $.013(.012)$ & $.014(.012)$ & $.007(.007)$ \\
$p T_{C} U_{O}$ & $.020(.024)$ & $.027(.041)$ & $.019(.025)$ \\
$p U_{C} T_{O}$ & $.022(.036)$ & $.030(.052)$ & $.025(.025)$ \\
$p N 1_{C} U_{O}$ & $.005(.008)$ & $.010(.013)$ & $.008(.013)$ \\
$p U_{C} N 1_{O}$ & $.005(.008)$ & $.012(.020)$ & $.004(.004)$ \\
$p U_{C} U_{O}$ & $.053(.063)$ & $.082(.107)$ & $.053(.071)$ \\
$\mu_{\mathrm{C}}$ & $1.00(5.55)$ & $0.16(6.23)$ & $1.20(6.75)$ \\
$\mu_{\mathrm{O}}$ & $0.17(2.05)$ & $-0.01(3.04)$ & $0.34(3.15)$ \\
$\sigma_{\mathrm{C}}$ & $21.90(6.37)$ & $24.35(11.55)$ & $21.20(7.20)$ \\
$\sigma_{\mathrm{O}}$ & $13.03(2.78)$ & $14.84(4.79)$ & $13.11(4.82)$ \\
\hline
\end{tabular}

Group means, with standard deviations presented in parentheses. $\mu_{\mathrm{C}}$ and $\sigma_{\mathrm{C}}$ range from $-180^{\circ}$ to $+180^{\circ}$, whereas $\mu_{\mathrm{O}}$ and $\sigma_{\mathrm{O}}$ range from $-90^{\circ}$ to $+90^{\circ}(\sigma=\sqrt{1 / \kappa})$

\section{Joint-modeling results}

Across all conditions, the vast majority of responses were attributed to reporting both features of the correct target item (correlated target responses), as reflected in the scatterplots as a central density of responses at the origin (see Fig. 2b) and the overall high proportion of $\mathrm{T}_{\mathrm{C}} \mathrm{T}_{\mathrm{O}}$ responses in Fig. 3. Critically, as predicted, the saccade manipulation degraded performance. Presenting the array $50 \mathrm{~ms}$ after a saccade resulted in greater feature errors, with a significantly lower probability of correlated target responses $\left(\mathrm{T}_{\mathrm{C}} \mathrm{T}_{\mathrm{O}}\right)$ in Saccade-Early trials compared with no-saccade trials, $t(24)=3.48, p=.002, d=$ 0.70 . However, when the array was presented $500 \mathrm{~ms}$ after a saccade, performance rebounded, with significantly greater correlated target responses in saccade-late trials compared with saccade-early trials, $t(24)=3.60, p=.001, d=0.72$, and no difference between saccade-late and no-saccade trials, $t(24)=0.02, p=.987, d<0.01$ (see Fig. 3a). The standard

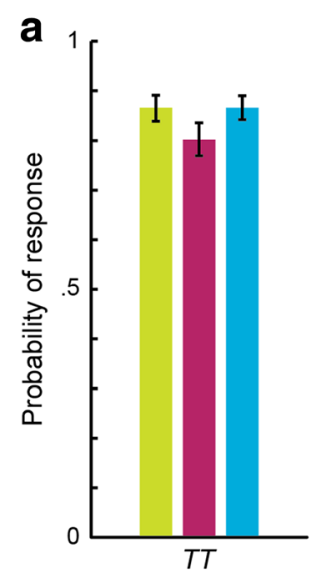

b

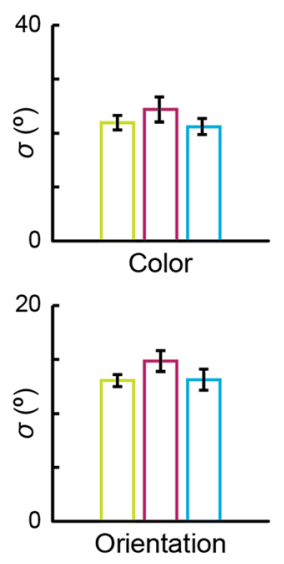

No-Saccade

Saccade-Early (50 ms)

Saccade-Late $(500 \mathrm{~ms})$
C

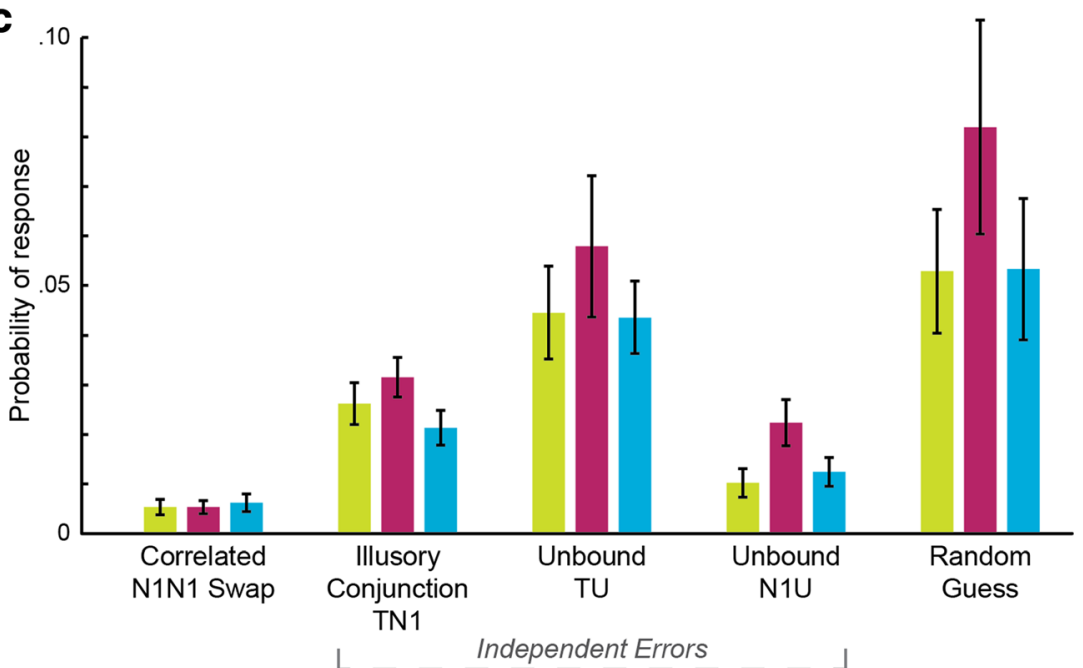

Fig. 3 Simple joint model maximum-likelihood parameter estimates. The graphs in (a) present group means of best-fit estimates for the probability of correlated target responses $\left(\mathrm{T}_{\mathrm{C}} \mathrm{T}_{\mathrm{O}}\right)$ and in $(\mathbf{b})$ the standard deviations of the $\mathrm{T}_{\mathrm{C}} \mathrm{T}_{\mathrm{O}}$ distribution for color $\left(\sigma_{\mathrm{C}}\right)$ and orientation $\left(\sigma_{\mathrm{O}}\right)$ for each condition. The graphs in (c) present the proportion of erroneous responses (i.e., non- $\mathrm{T}_{\mathrm{C}} \mathrm{T}_{\mathrm{O}}$ ) that can be attributed to correlated $\mathrm{N}_{\mathrm{C}} \mathrm{N} 1_{\mathrm{O}}$ swaps; different types of independent errors: illusory conjunctions (e.g., $\mathrm{T}_{\mathrm{C}} \mathrm{N} 1_{\mathrm{O}}$ ), unbound targets (e.g., $\mathrm{T}_{\mathrm{C}} \mathrm{U}_{\mathrm{O}}$ ), and unbound nontargets (e.g., $\mathrm{N}_{\mathrm{C}} \mathrm{U}_{\mathrm{O}}$ ); and random guesses. Error bars represent standard errors 
Table 3 Summary of comparisons for simple model response types and parameters

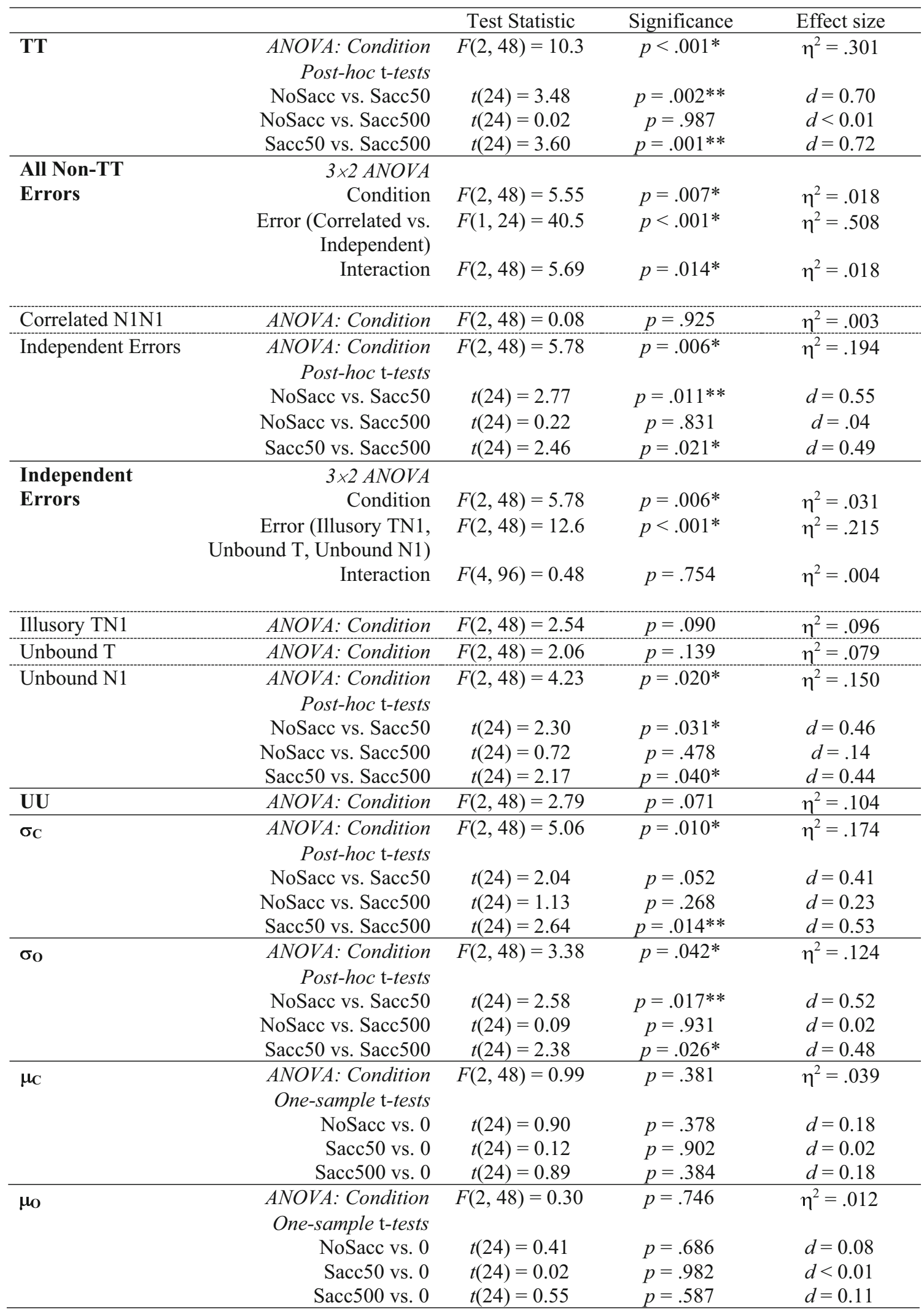

Post hoc $t$ tests reported only for significant main effects. ${ }^{*} p<.05$.

$* * p<.017$ (Bonferroni-corrected for multiple post hoc comparisons) 
deviations of both color $\left(\sigma_{\mathrm{C}}\right)$ and orientation $\left(\sigma_{\mathrm{O}}\right)$ responses were also greater for saccade-early trials than for the other conditions (see Fig. 3b; see Table 3 for all comparisons), indicating less precise feature reports when spatial attention was still updating immediately after a saccade. The performance drop for saccade-early trials was not simply because the task was too hard; random guessing (i.e., $\mathrm{U}_{\mathrm{C}} \mathrm{U}_{\mathrm{O}}$ ) was only marginally significantly different across conditions, $F(2,48)=2.79$, $p=.071, \eta^{2}=.03$. Although a number of factors could contribute to an increase in random guessing or a decrease in precision immediately after a saccade, including increased task difficulty, noisy encoding, and/or attentional resources at the saccade landing point (e.g., Schneider, 2013), a benefit of our joint-feature model is that we can explore specific types of feature-binding errors on top of these more general performance indicators.

Our critical question was, What types of binding errors occur when spatial attention is dynamically updating immediately after a saccade? One hypothesis is that remapping is simply a single-spotlight process of shifting attention that occurs with variable latency (such that on some trials attention has already updated to the spatiotopic, T, location, and on others it is still stuck at the initial retinotopic, N1, location)which should primarily produce greater correlated $\mathrm{N}_{\mathrm{C}} \mathrm{N} 1_{\mathrm{O}}$ swap errors in saccade-early trials, similar to the shift condition of Dowd and Golomb (2019). However, we hypothesize that remapping instead occurs in two temporally overlapping stages (see Golomb, 2019), such that spatial attention is transiently split across the spatiotopic target (T) location and the retinotopic trace $(\mathrm{N} 1)$ location immediately after the saccade - which should produce greater independent errors in saccade-early trials, similar to the split condition of Dowd and Golomb (2019).

We first analyzed simple model parameter estimates with a broad repeated-measures ANOVA across error type (correlated $\mathrm{N} 1_{\mathrm{C}} \mathrm{N} 1_{\mathrm{O}}$ swaps vs. all independent responses; see Table 1) and condition (no-saccade, saccade-early, saccade-late conditions), revealing a significant interaction effect, $F(2,48)=5.69, p=.006, \eta^{2}=.01$. Thus, we followed up with two separate repeated-measures ANOVAs across condition, first for correlated $\mathrm{N}_{\mathrm{C}} \mathrm{N} 1_{\mathrm{O}}$ swaps, $F(2,48)=0.08, p=$ $.925, \eta^{2}<.01$, and then for all independent responses, $F(2,48)$ $=5.78, p=.006, \eta^{2}=.04$. As illustrated in Fig. 3c, correlated $\mathrm{N} 1_{\mathrm{C}} \mathrm{N} 1_{\mathrm{O}}$ swap errors were overall rare and not significantly different across conditions. However, independent errors were significantly more frequent in saccade-early compared with no-saccade trials, $t(24)=2.77, p=.011, d=0.55$, and marginally more frequent in saccade-early compared with saccade-late trials, $t(24)=2.46, p=.021, d=0.49$ (compared with Bonferroni-corrected thresholds of $p=.017$ ).

To deconstruct further, we then examined whether the saccade-early increase in independent errors was attributable to increased illusory conjunctions (e.g., $\mathrm{T}_{\mathrm{C}} \mathrm{N} 1_{\mathrm{O}}$ ), unbound targets (e.g., $\mathrm{U}_{\mathrm{C}} \mathrm{T}_{\mathrm{O}}$ ), and/or unbound nontargets (e.g., $\mathrm{N1}_{\mathrm{C}} \mathrm{U}_{\mathrm{O}}$ ).
Previous single-feature studies demonstrated that in the critical period immediately after a saccade, participants produced more "mixing" errors, or a blending between the spatiotopic target and retinotopic distractor colors (Golomb et al., 2014). Although we found no evidence of mixing within feature dimensions here (means $\mu_{\mathrm{C}}$ and $\mu_{\mathrm{O}}$ of the target were not different from zero nor different across conditions, $p \mathrm{~s}>.38$; see Table 3), illusory conjunctions could potentially be thought of as participants producing mixing across featuredimensions - such that they "misbind" one feature of the target with one feature of the retinotopic distractor (i.e., an illusory conjunction between $\mathrm{T}$ and N1). The other types of independent errors can be thought of as "unbinding" (i.e., reporting only one feature of one item). A repeatedmeasures ANOVA across condition and independent error type (illusory conjunction, unbound target, unbound nontarget) revealed a significant main effect of condition, $F(2,48)=$ 5.78, $p=.006, \eta^{2}=.02$ (identical to the one-way ANOVA from before), and a significant effect of error type, $F(2,48)=$ 12.6, $p<.001, \eta^{2}=.14$, revealing that across all conditions, unbound targets occurred more often than illusory conjunctions or unbound nontargets. However, there was not a significant interaction effect, $F(4,96)=0.48, p=.754, \eta^{2}<.01$ (see Fig. 3c). The lack of an interaction suggests that the increase in independent errors for saccade-early trials was not driven more by one subtype of independent error than the others. Thus, although the pattern of results supports an increase in illusory conjunctions (i.e., mixing across feature dimensions), increased independent errors immediately after a saccade are not driven by illusory conjunctions alone; instead, the results suggest that dynamic spatial remapping after a saccade leads to a breakdown of object-feature binding more generally.

The simple model used to generate the results above assumes that independent errors stem from reporting a single feature from the target or the retinotopic N1 nontarget. Given our theoretical focus on remapping spatial attention across these two critical locations, illusory conjunctions were defined in the simple model as binding between spatiotopic $\mathrm{T}$ and retinotopic $\mathrm{N} 1$ items specifically (e.g., $\mathrm{T}_{\mathrm{C}} \mathrm{N} 1_{\mathrm{O}}$ ). But were illusory conjunctions between $\mathrm{T}$ and the retinotopic $\mathrm{N} 1$ distractor actually more likely than between $\mathrm{T}$ and the control $\mathrm{N} 2$ distractor? To confirm that the retinotopic N1 location was indeed the critical nontarget that triggered illusory conjunctions, we used the supplemental full model parameter fits to compare across combinations of $\mathrm{T}$ with all of the nontarget items (e.g., $\mathrm{T}_{\mathrm{C}} \mathrm{N} 1_{\mathrm{O}}, \mathrm{T}_{\mathrm{C}} \mathrm{N} 2_{\mathrm{O}}, \mathrm{T}_{\mathrm{C}} \mathrm{N} 3_{\mathrm{O}}$ ). For saccade-early trials, participants were numerically more likely to report illusory conjunctions between $\mathrm{T}$ and $\mathrm{N} 1$ items $\left(p T_{C} N 1_{O}+p N 1_{C} T_{O}=\right.$ $.042,95 \%$ HDI $[.031, .056])$ than illusory conjunctions between $\mathrm{T}$ and $\mathrm{N} 2$ items $\left(p T_{C} N 2_{O}+p N 2_{C} T_{O}=.028[.017\right.$, $.038]$ ), with only a slight overlap between their 95\% HDIs (full model parameter estimates were considered significantly different if their 95\% HDIs did not overlaps; Kruschke, 2011). 


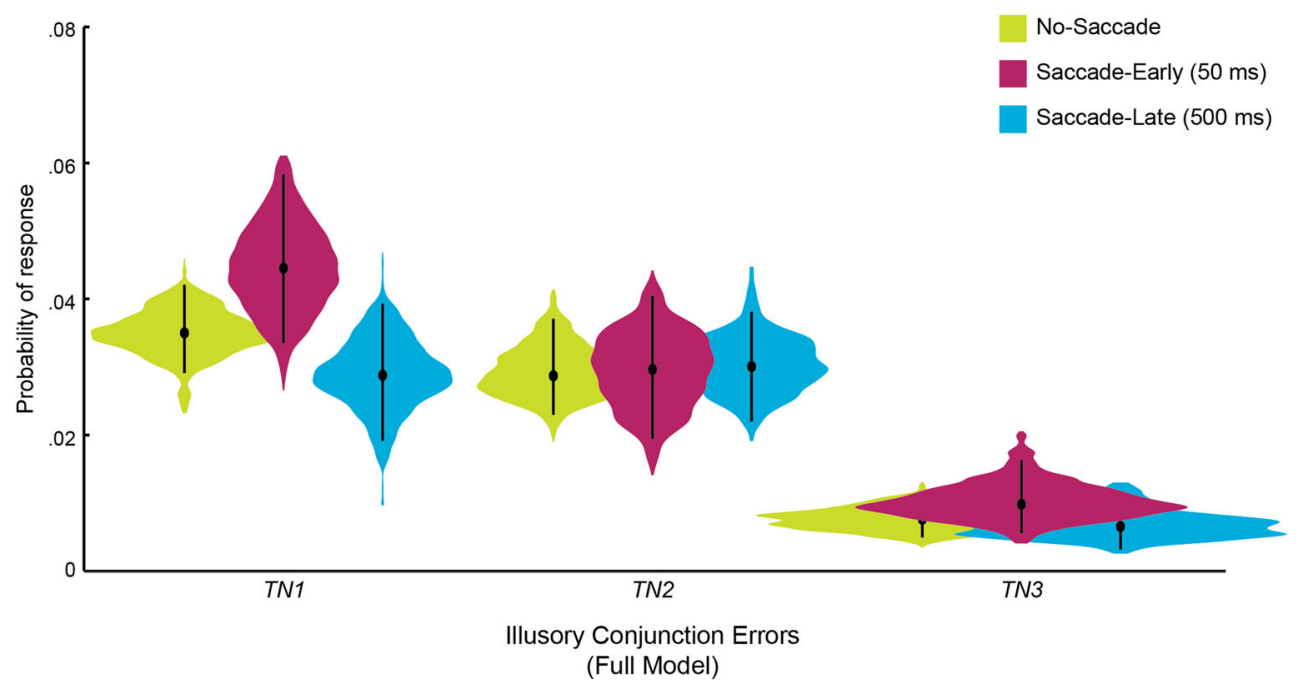

Fig. 4 Full joint model maximum a posteriori estimates for independent target errors suggest that the retinotopic N1 distractor specifically interfered with performance. For each response type (i.e., combinations of $T$ with each nontarget), a violin plot illustrates the posterior distribution of each parameter over 15,000 postconvergence samples. The black dots

In contrast, in no-saccade and saccade-late trials, the TN1 and TN2 probabilities were nearly equal (see Fig. 4; HDIs overlap almost entirely).

\section{Discussion}

Our perception of visual stability is facilitated by the dynamic remapping of visual information across eye movements (e.g., Duhamel et al., 1992), but several recent papers have suggested this process is not as rapid or efficient as previously thought (e.g., Golomb et al., 2008; Golomb et al., 2010; Mathôt \& Theeuwes, 2010). Here, we examined how the remapping of spatial attention across saccades affects visual object integrity.

We tested the hypothesis that, for a brief period of time after each eye movement, spatial attention lingers at the previous retinotopic location (e.g., Golomb et al., 2008; Golomb et al., 2010), such that attention is transiently split across the correct spatiotopic location and the retinotopic trace location. Previous work has demonstrated that in the absence of eye movements, splitting covert spatial attention across multiple locations degrades object integrity, whereas rapid shifts of spatial attention maintain bound objects (Dowd \& Golomb, 2019). In the current pre-registered study, participants were not explicitly attending to two different locations, but rather were told to maintain attention at a single spatiotopic location across a saccade. Nevertheless, we found that immediately after a saccadic eye movement, participants made more erroneous feature reports. It was not simply that participants randomly guessed more (marginal increase in $U_{C} U_{O}$ responses immediately after a saccade), but specifically, participants mark each parameter's best-fit estimate, and the whiskers represent the 95\% highest density interval. The full model was fit for each condition (upper-right legend) separately, collapsed across all subjects. See Supplement S1 for more information

reported more independent feature errors (i.e., illusory conjunctions, unbound target, and unbound nontarget responses) - as if spatial attention were indeed briefly split across multiple locations on some trials.

These failures of object-feature binding after a saccade were both temporally and spatially specific. The increase in independent errors was present only when objects were presented a short interval $(50 \mathrm{~ms})$ after each eye movement; performance rebounded when the objects were presented after a longer postsaccadic delay $(500 \mathrm{~ms})$, consistent with a shortlived "retinotopic attentional trace" that decays after $\sim 150 \mathrm{~ms}$ (Golomb et al., 2008). The errors themselves also seemed to reflect interference from residual attention at another (i.e., the retinotopic trace) location: The increase in independent errors included both unbinding (i.e., reporting only one feature of either the spatiotopic $\mathrm{T}$ or retinotopic N1 item; unbound errors) and misbinding (i.e., associating one feature of the spatiotopic $\mathrm{T}$ item with the other feature of the retinotopic $\mathrm{N} 1$ item; illusory conjunctions) of features. In the supplementary Full Model analyses, illusory conjunctions were also numerically more likely for the spatiotopic $\mathrm{T}$ and retinotopic $\mathrm{N} 1$ items than for the spatiotopic $\mathrm{T}$ and equidistant control $\mathrm{N} 2$ items, supporting the idea of spatial interference by residual retinotopic attention.

One initial hypothesis was that residual retinotopic attention during dynamic remapping would result in an increase in illusory conjunctions between spatiotopic $\mathrm{T}$ and retinotopic $\mathrm{N} 1$ items. However, we found that that the increase in independent errors immediately after a saccade was not driven by these illusory conjunctions alone. Instead, increased independent errors may have been more reflective of general unbinding, which mimics the pattern found when participants 
intentionally and covertly split attention between two cued spatial locations (split vs. hold conditions of Dowd \& Golomb, 2019) - in that study, splitting attention across two locations also resulted in greater independent errors compared to maintaining attention at one location, and this increase was significantly driven by unbound targets rather than illusory conjunctions or unbound nontargets. Taken together, these results suggest that dynamic spatial remapping after a saccade is akin to splitting covert attention in that it leads to a general breakdown of object-feature binding.

These results demonstrate perceptual consequences consistent with a two-stage, or dual-spotlight (Golomb, 2019), model of remapping, in which "turning on" of the new retinotopic location is distinct from "turning off" the previous retinotopic location, allowing for a brief window of time during which both locations are simultaneously attended (e.g., Golomb et al., 2011). Previous studies have demonstrated systematic distortions within a single feature dimension immediately after an eye movement (e.g., reporting the blending of colors at spatiotopic $\mathrm{T}$ and retinotopic N1 locations; Golomb et al., 2014). By highlighting the integration of multiple features into objects, the current results provide yet more support that remapping of attention across eye movements results in a temporary splitting of attention across both locations. An alternative single-stage process of remapping would predict that a single focus of spatial attention shifts from one location to another, such that at a given moment, attention is either at the updated (spatiotopic) location or stuck at the initial (retinotopic trace) location. If this were the case, we would expect remapping to involve primarily a shift of attention, which should preserve object integrity in this multifeature paradigm (as in the exogenously induced shift condition of Dowd \& Golomb, 2019). In other words, a simple shift of attention would result in a mixture of some trials in which attention had successfully remapped (resulting in correlated target $T_{C} T_{O}$ responses) and some trials in which attention was still at the previous retinotopic location (resulting in correlated $\mathrm{N}_{\mathrm{C}} \mathrm{N}_{\mathrm{O}}$ swap errors). However, in the current study, there was no increase in correlated $\mathrm{N}_{\mathrm{C}} \mathrm{N} 1_{\mathrm{O}}$ swap errors immediately after a saccade. Moreover, if the speed of such a hypothetical remapping shift was faster than an exogenously induced shift (i.e., Dowd \& Golomb, 2019), then attention should have already finished remapping to the new retinotopic location by the time the array was presented, such that there would be similar rates of correlated $T_{C} T_{O}$ responses immediately and later after a saccade - which was not the case. Another alternative single spotlight (i.e., serial) model might posit that attention shifts rapidly back and forth between the previous retinotopic and new retinotopic locations, with limited attention at either location (cf. Jans, Peters, \& De Weerd, 2010), resulting in an increase in independent errors. However, it is unclear how this back-and-forth account would apply to dynamic remapping after a saccade, where there are clear priorities in retinotopic space (i.e., from trace to new). Instead, the increase in independent errors suggests that spatial attention was transiently split across multiple locations, within a single trial, similar to the covert attention split condition of Dowd and Golomb (2019).

It should be noted that the present study examines "feature integration" in the sense of Treisman's feature integration theory (Treisman \& Gelade, 1980) - the idea that spatial attention serves as the "glue" that binds different features of an object together, when multiple objects are simultaneously present in the visual field. Thus, the goal was to test how dynamic spatial attention induced by a saccade influences object-feature binding, for multifeature stimuli presented immediately after an eye movement. This is not to be mistaken with transsaccadic feature integration (integrating features presented at different points in time before, during, and after an eye movement; e.g., blending of presaccadic and postsaccadic orientation or color; Melcher, 2005; Oostwoud Wijdenes, Marshall, \& Bays, 2015). Our question here is not how features are perceptually integrated over time across an eye movement, nor whether an object presented prior to an eye movement preserves its integrity across the saccade (e.g., Hollingworth, Richard, \& Luck, 2008; Shafer-Skelton, Kupitz \& Golomb, 2017), but rather how the process of attentional remapping across a saccade impacts object-feature binding. Nevertheless, it is interesting to note that previous studies have induced the misbinding or unbinding of features by breaking transsaccadic object correspondence, such that attention might be split between separate presaccadic and postsaccadic object representations (Poth, Herwig, \& Schneider, 2015). Compare that with the current study, in which misbinding and unbinding of features arise for objects presented immediately after a saccade, such that attention might be split between separate spatial locations. Here, the process of dynamically remapping attention presents yet another instance of the binding problem (Treisman, 1996; Wolfe \& Cave, 1999).

Treisman's initial concept of linking multiple feature dimensions via spatial attention (Treisman \& Gelade, 1980) has been extended into a multitude of studies that argue that spatial location serves as the anchor for objectfeature binding (e.g., Nissen, 1985; Pertzov \& Husain, 2013; Reynolds \& Desimone, 1999; Schneegans \& Bays, 2017; Vul \& Rich, 2010; see also Schneegans \& Bays, 2018). But such space-binding models do not necessarily account for changing spatial reference frames (i.e., retinotopic vs. spatiotopic) across eye movements (e.g., Schneegans \& Bays, 2017; see also Cavanagh, Hunt, Afraz, \& Rolfs, 2010). The current findings demonstrate that even when only one spatiotopic location is task relevant, dynamic remapping across eye movements can produce, even transiently, multiple foci of attention, and this can result in a breakdown of object integrity. We speculate that this breakdown is a bug rather than a feature of the visual system, such that when our attention is 
overly taxed, there may be suboptimal consequences for visual perception. The disruption of object-feature binding when attention is remapped and when spatial reference frames are updated thus poses a unique challenge for models of object-feature integration. Nevertheless, the current results also more broadly emphasize the importance of a single locus of spatial attention for intact object integrity (see also Dowd \& Golomb, 2019). Overall, these failures of object integrity after eye movements not only underline the importance of spatial attention but also suggest how vulnerable object perception may be in the real world, when our eyes are constantly moving across multiple objects with multiple features in the environment.

Open practices statement The participant recruitment techniques, target sample size, exclusion rules, stimuli, task design and procedure, and statistical models and analyses were preregistered on the Open Science Framework (http://osf.io/ y495a) prior to data collection. Materials are available on OSF (https://osf.io/btsyz/).

Acknowledgements We thank Alexandra Haeflein, Chris Jones, Robert Murcko, and Samoni Nag for assistance with data collection.

Funding This study was funded by grants from the National Institutes of Health (R01-EY025648 to J. D. Golomb, F32-028011 to E. W. Dowd) and the Alfred P. Sloan Foundation (to J. D. Golomb).

\section{References}

Bays, P. M. (2016). Evaluating and excluding swap errors in analogue tests of working memory. Nature Publishing Group, 1-14. doi: https://doi.org/10.1038/srep19203

Bays, P. M., Catalao, R. F. G., \& Husain, M. (2009). The precision of visual working memory is set by allocation of a shared resource. Journal of Vision, 9(10), 7. doi:https://doi.org/10.1167/9.10.7

Bays, P. M., Wu, E. Y., \& Husain, M. (2011). Storage and binding of object features in visual working memory. Neuropsychologia, 49(6), 1622-1631. doi:https://doi.org/10.1016/j.neuropsychologia.2010. 12.023

Cavanagh, P., Hunt, A. R., Afraz, A., \& Rolfs, M. (2010). Visual stability based on remapping of attention pointers. Trends in Cognitive Sciences, 14(4), 147-153. doi:https://doi.org/10.1016/j.tics.2010. 01.007

Cornelissen, F. W., Peters, E. M., \& Palmer, J. (2002). The Eyelink Toolbox: Eye tracking with MATLAB and the Psychophysics Toolbox. Behavior Research Methods, Instruments, \& Computers, 34, 613-617.

Dowd, E. W., \& Golomb, J. D. (2019). Object-feature binding survives dynamic shifts of spatial attention. Psychological Science, 30(3), 343-361. doi:https://doi.org/10.1177/0956797618818481

Duhamel, J. R., Colby, C. L., \& Goldberg, M. E. (1992). The updating of the representation of visual space in parietal cortex by intended eye movements. Science, 255(5040), 90-92. doi:https://doi.org/10. $1126 /$ science. 1553535

Duncan, J. (1984). Selective attention and the organization of visual information. Journal of Experimental Psychology: General, 113(4), 501-517. doi:https://doi.org/10.1037/0096-3445.113.4.501

Gelman, A., \& Rubin, D. B. (1992). Inference from iterative simulation using multiple sequences. Statistical Science, 7(4), 457-472. doi: https://doi.org/10.1214/ss/1177011136

Golomb, J. D. (2015). Divided spatial attention and feature-mixing errors. Attention, Perception, \& Psychophysics, 77(8), 2562-2569. doi: https://doi.org/10.3758/s13414-015-0951-0

Golomb, J. D. (2019). The what and when of remapping across saccades: a dual-spotlight theory of attentional updating and its implications for feature perception. Current Opinion in Psychology. doi:https:// doi.org/10.1016/j.copsyc.2019.03.018

Golomb, J. D., Chun, M. M., \& Mazer, J. A. (2008). The native coordinate system of spatial attention is retinotopic. Journal of Neuroscience, 28(42), 10654-10662. doi:https://doi.org/10.1523/ JNEUROSCI.2525-08.2008

Golomb, J. D., L'Heureux, Z. E., \& Kanwisher, N. (2014). Featurebinding errors after eye movements and shifts of attention. Psychological Science, 25(5), 1067-1078. doi:https://doi.org/10. 1177/0956797614522068

Golomb, J. D., Marino, A. C., Chun, M. M., \& Mazer, J. A. (2011). Attention doesn't slide: Spatiotopic updating after eye movements instantiates a new, discrete attentional locus. Attention, Perception, \& Psychophysics, 73(1), 7-14. doi:https://doi.org/10.3758/s13414010-0016-3

Golomb, J. D., Nguyen-Phuc, A. Y., Mazer, J. A., McCarthy, G., \& Chun, M. M. (2010). Attentional facilitation throughout human visual cortex lingers in retinotopic coordinates after eye movements. Journal of Neuroscience, 30(31), 10493-10506. doi:https://doi.org/10.1523/ JNEUROSCI.1546-10.2010

Hollingworth, A., Richard, A. M., \& Luck, S. J. (2008). Understanding the function of visual short-term memory: Transsaccadic memory, object correspondence, and gaze correction. Journal of Experimental Psychology: General, 137(1), 163-181. doi:https:// doi.org/10.1037/0096-3445.137.1.163

Jans, B., Peters, J. C., \& De Weerd, P. (2010). Visual spatial attention to multiple locations at once: The jury is still out. Psychological Review, 117, 637-682. doi:https://doi.org/10.1037/a0019082

Jonikaitis, D., Szinte, M., Rolfs, M., \& Cavanagh, P. (2013). Allocation of attention across saccades. Journal of Neurophysiology, 109(5), 1425-1434. doi:https://doi.org/10.1152/jn.00656.2012

Khayat, P. S., Spekreijse, H., \& Roelfsema, P. R. (2006). Attention lights up new object representations before the old ones fade away. Journal of Neuroscience, 26(1), 138-142. doi:https://doi.org/10. 1523/JNEUROSCI.2784-05.2006

Kleiner, M., Brainard, D., \& Pelli, D. (2007). What's new in Psychtoolbox-3? Perception, 36(14), 1-16. (ECVP Abstract Supplement)

Kruschke, J. K. (2011). Bayesian assessment of null values via parameter estimation and model comparison. Perspectives on Psychological Science, 6(3), 299-312. doi:https://doi.org/10.1177/ 1745691611406925

Mathôt, S., \& Theeuwes, J. (2010). Gradual remapping results in early retinotopic and late spatiotopic inhibition of return. Psychological Science, 21(12), 1793-1798. doi:https://doi.org/10.1177/ 0956797610388813

Melcher, D. (2005). Spatiotopic transfer of visual-form adaptation across saccadic eye movements. Current Biology, 15(19), 1745-1748. doi: https://doi.org/10.1016/j.cub.2005.08.044

Nissen, M. J. (1985). Accessing features and objects: Is location special? In M. I. Posner \& O. S. Marin (Eds.), Attention and performance XI (pp. 205-219). London, UK: Routledge. doi:https://doi.org/10. $4324 / 9781315630236$ 
O’Craven, K. M., Downing, P. E., \& Kanwisher, N. (1999). fMRI evidence for objects as the units of attentional selection. Nature, 401(6753), 584-587. doi:https://doi.org/10.1038/44134

Oostwoud Wijdenes, L., Marshall, L., \& Bays, P. M. (2015). Evidence for optimal integration of visual feature representations across saccades. Journal of Neuroscience, 35(28), 10146-10153. doi:https://doi.org/ 10.1523/jneurosci.1040-15.2015

Pertzov, Y., \& Husain, M. (2013). The privileged role of location in visual working memory. Attention, Perception, \& Psychophysics, 76(7), 1914-1924. doi:https://doi.org/10.3758/s13414-013-0541-y

Poth, C. H., Herwig, A., \& Schneider, W. X. (2015). Breaking object correspondence across saccadic eye movements deteriorates object recognition. Frontiers in System Neurosciences, 9, 176. doi:https:// doi.org/10.3389/fnsys.2015.00176

Reynolds, J. H., \& Desimone, R. (1999). The role of neural mechanisms of attention in solving the binding problem. Neuron, 24(1), 19-29. doi:https://doi.org/10.1016/s0896-6273(00)80819-3

Rolfs, M., Jonikaitis, D., Deubel, H., \& Cavanagh, P. (2011). Predictive remapping of attention across eye movements. Nature Neuroscience, 14(2), 252-256. doi:https://doi.org/10.1038/nn.2711

Schneegans, S., \& Bays, P. M. (2017). Neural architecture for feature binding in visual working memory. Journal of Neuroscience, 37(14), 3913-3925. doi:https://doi.org/10.1523/JNEUROSCI. 3493-16.2017

Schneegans, S., \& Bays, P. M. (2018). New perspectives on binding in visual working memory. British Journal of Psychology. Advance online publication. doi:https://doi.org/10.1111/bjop.12345

Schneider, W. X. (2013). Selective visual processing across competition episodes: A theory of task-driven visual attention and working memory. Philosophical Transactions of the Royal Society B: Biological Sciences, 368(1628), 20130060. doi:https://doi.org/10. 1098/rstb.2013.0060

Schoenfeld, M. A., Tempelmann, C., Martinez, A., Hopf, J. M., Sattler, C., Heinze, H. J., \& Hillyard, S. A. (2003). Dynamics of feature binding during object-selective attention. Proceedings of the National Academy of Sciences, 100(20), 11806-11811. doi: https:// doi.org/10.1073/pnas.1932820100
Shafer-Skelton, A., Kupitz, C. N., \& Golomb, J. D. (2017). Objectlocation binding across a saccade: A retinotopic spatial congruency bias. Attention, Perception, \& Psychophysics, 79(3), 765-781. doi: https://doi.org/10.3758/s13414-016-1263-8

Suchow, J. W., Brady, T. F., Fougnie, D., \& Alvarez, G. A. (2013). Modeling visual working memory with the MemToolbox. Journal of Vision, 13(10), 9. doi:https://doi.org/10.1167/13.10.9

Talsma, D., White, B. J., Mathôt, S., Munoz, D. P., \& Theeuwes, J. (2013). A retinotopic attentional trace after saccadic eye movements: Evidence from event-related potentials. Journal of Cognitive Neuroscience, 25(9), 1563-1577. doi:https://doi.org/10. 1162/jocn_a_00390

Treisman, A. (1996). The binding problem. Current Opinion in Neurobiology, 6(2), 171-178. doi:https://doi.org/10.1016/S09594388(96)80070-5

Treisman, A. M., \& Gelade, G. (1980). A feature-integration theory of attention. Cognitive Psychology, 12(1), 97-136.

Treisman, A., \& Schmidt, H. (1982). Illusory conjunctions in the perception of objects. Cognitive Psychology, 14, 107-141. doi:https://doi. org/10.1093/acprof:osobl/9780199734337.003.0019

Vul, E., \& Rich, A. N. (2010). Independent sampling of features enables conscious perception of bound objects. Psychological Science, 21(8), 1168-1175. doi:https://doi.org/10.1177/0956797610377341

Wilken, P., \& Ma, W. J. (2004). A detection theory account of change detection. Journal of Vision, 4(12), 1120-1135. doi:https://doi.org/ $10.1167 / 4.12 .11$

Wolfe, J. M., \& Cave, K. R. (1999). The psychophysical evidence for a binding problem in human vision. Neuron, 24(1), 11-17. doi:https:// doi.org/10.1016/s0896-6273(00)80818-1

Zhang, W., \& Luck, S. J. (2008). Discrete fixed-resolution representations in visual working memory. Nature, 453(7192), 233-235. doi:https:// doi.org/10.1038/nature06860

Publisher's note Springer Nature remains neutral with regard to jurisdictional claims in published maps and institutional affiliations. 